\title{
Mild Cardiac Valve Stenosis
}

National Cancer Institute

\section{Source}

National Cancer Institute. Mild Cardiac Valve Stenosis. NCI Thesaurus. Code C99986.

The cardiac valve orifice is abnormally narrow, to a mild degree. (ACC) 